\title{
Image Analysis Techniques for Ripeness Detection of Palm Oil Fresh Fruit Bunches
}

\author{
Shuwaibatul Aslamiah Ghazalli ${ }^{1 *}$, Hazlina Selamat ${ }^{2}$, Zaid Omar $^{3}$, Rubiyah Yusof ${ }^{4}$ \\ ${ }^{1}$ Malaysia-Japan International Institute of Technology, Universiti Teknologi Malaysia, Jalan Sultan Yahya Petra, 54100, \\ Kuala Lumpur, Malaysia \\ ${ }^{2}$ Centre for Artificial Intelligence and Robotics, School of Electrical Engineering, Universiti Teknologi Malaysia, 81310, \\ Johor Bahru, Johor, Malaysia \\ ${ }^{3}$ School of Electrical Engineering, Universiti Teknologi Malaysia, 81310 UTM Skudai, Johor, Malaysia. \\ ${ }^{4}$ Centre for Artificial Intelligence and Robotics, Universiti Teknologi Malaysia 54100, Kuala Lumpur Malaysia \\ *Corresponding author: hazlina@utm.my, Tel: 607-5535324, Fax: 607-5566272
}

\begin{abstract}
Being one of the biggest producers and exporters of palm oil and palm oil products, Malaysia has an important role to play in fulfilling the growing global need for oils and fats sustainably. Quality is an important factor that ensuring palm oil industries fulfill the demands of palm oil product. There has significant relationship between the quality of the palm oil fruits and the content of its oil. Ripe FFB gives more oil content, while unripe FFB give the least content. Overripe FFB shows that the content of oil is deteriorates. There have 4 classes of ripeness stages involves in this paper which are ripe, unripe, underipe and overripe. The proposed approach in this paper uses color features and bag of visual word for classifying oil palm fruit ripeness stages. Experiments conducted in this paper consisted of smartphone camera for image acquisition, python and matlab software for image pre processing and Support Vector Machine for classification. A total of 400 images is taken in a few plant in north Malaysia. Experiments involved on a dataset of 360 images for training for four classes and 40 images for testing. The average accuracy for the 4 classes of the FFB by color features is $57 \%$ while the accuracy for ripeness classification by using bag of visual word is $70 \%$.
\end{abstract}

Keywords: image processing, classification, bag of visualword, SVM

Article History: received 13 September 2019; accepted 5 December 2019; published 18 December 2019.

(C) 2019 Penerbit UTM Press. All rights reserved

\section{INTRODUCTION}

Fresh Fruit Bunch (FFB) or known as Elaeisguineensisjacq originally come from West Africa where it grows in the wild and later was developed into an agricultural crop. FFB is an important agricultural commodity that has high production level. Various products produced from FFB are used worldwide in daily routine such as margarine, soap, cooking oil and more. Known as one of the biggest producers who make palm oil industry as their economic backbone, Malaysia have to improve the production flow in order to fulfill the world demands. Quality is the foremost imperative figure for oil palm industries since it is believed that quality is the most key to successful in today's competitive market. A research had been done by [1][2] to study the relationship between oil content and the quality of the FFB. This research show that high quality of FFB has the highest oil content while low quality of FFB has the least oil content. In [3] reported that there have 3 factor that lead to quality of palm oil which are ripeness of FFB, low free of fatty acid and high oil extraction rate. Since ripeness is seen by the clients as the most quality indicator of the fruits [3], monitoring and controlling produce FFB ripeness has gotten to be a critical issue within the crops industry. In order to determine the ripeness of FFB, appearance was used as main point to determine its maturity. Efficient and systematic procedure in palm oil industry may ease the process of producing high quality of palm oil.

At present, in palm oil industry, the ripeness of the FFB was based on manual sight inspection only[4]. It is made by palm oil industry worker or known as human grader by using their sight. Human mistake in manual visual inspection might be happened which will lead to cost loss. They were effortlessly affected by other components, actuating subjective and inconsistent evaluation results [3]. The effect of the inconsistencies of these inspection also will lead to the yield gap of palm oil in production [3]. The procedure of teaching up experienced staff in performing the task is not easy. They have to attend special class by the Malaysian Palm Oil Board (MPOB) and get the certificate to be a certified grader. According to MPOB guideline, the ripeness of the FFB was determine by its surface color and number of loose fruits detached from its palm[5]. MPOB has classify sixteen classes of FFB in palm oil mills which are ripe, underripe, unripe, overripe, empty, rotten, wet, long stalk, unfresh/old, dirty, small, pest damaged, diseased, dura, small stalk, and partenokarpi. Each of these classes have their own physical condition and only ripe FFB was high quality and accepted inmill without penalty[5]. The rest classes of the FFB either being charged with penalty or has low quality. However, most studies focussed on three to four stage of 
FFB only which are ripe, underripe, overripe and unripe[6]. Ripe FFB has Reddish Orange colour and must have 10 loose fruit detached from its palm, underripe FFB has semi orange color, unripe FFB has dark purple color and overripe FFB has 25-50\% loose fruit detached from its palm.[5] Figure 1, 2, 3 and 4 shown the ripe, underripe, overripe and unripe stage of ripeness respectively.

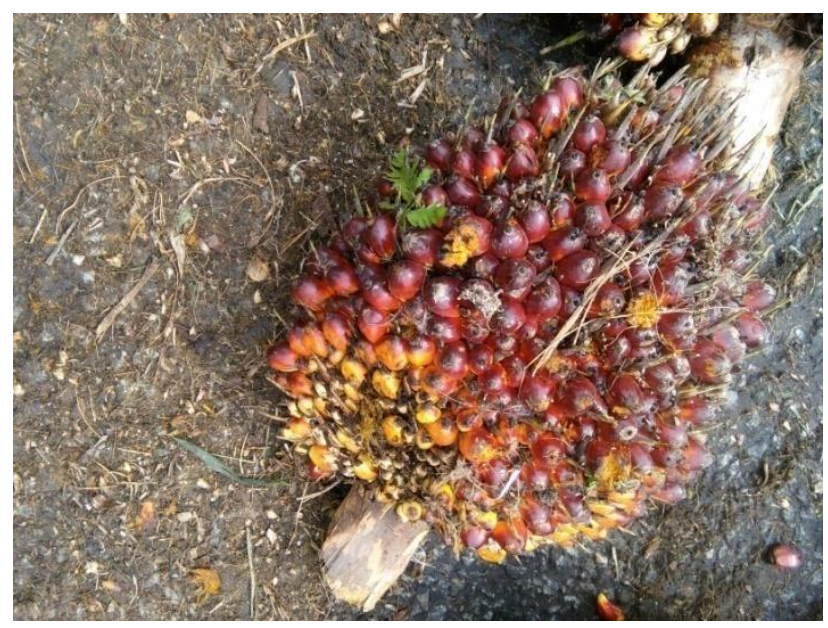

Figure 1. Ripe FFB

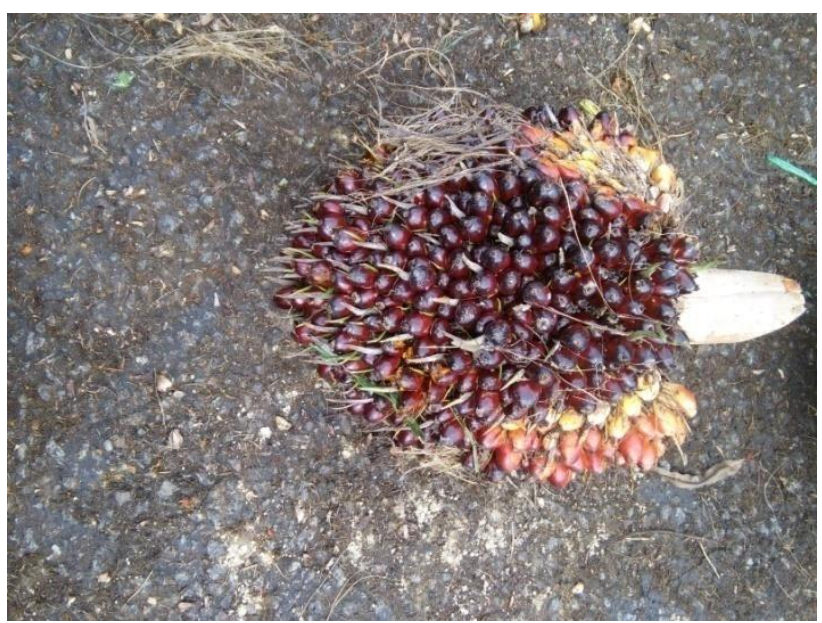

Figure 2. Underipe FFB

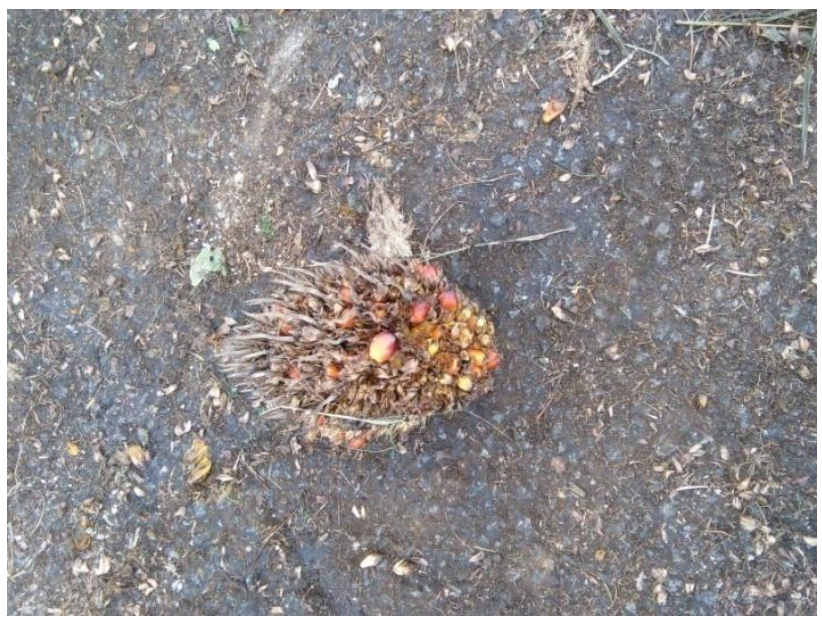

Figure 3. Overipe FFB

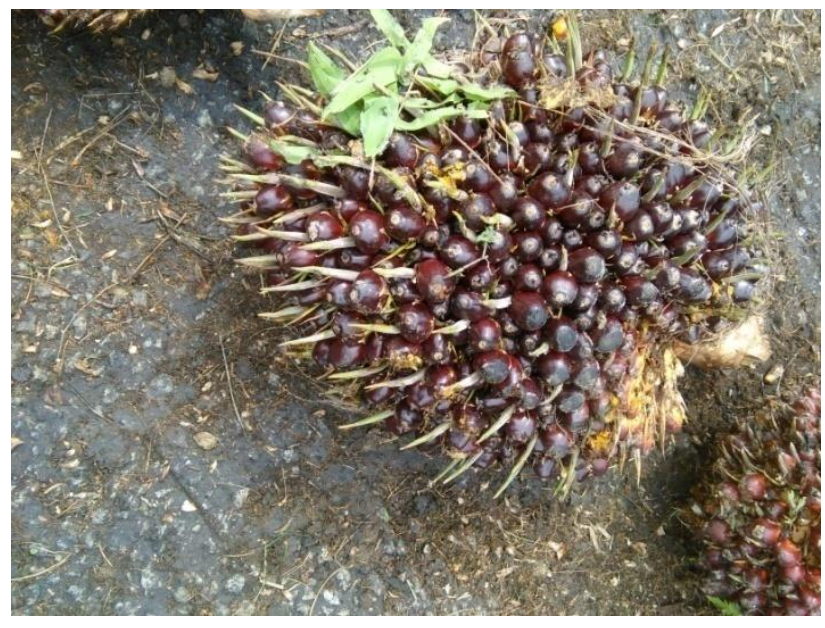

Figure 4. Unripe FFB

\section{LITERATURE REVIEW}

Since the innovation of computers, a lot of efforts have been made in order to make them learn. Efficient and systematic procedure in palm oil industry may ease the process of producing high quality of palm oil. For the past few years, machine vision applications for automated inspection and sorting of fruits and vegetables have been discussed and reported[7]. In [8], they analyzed the RGB and HSI data of the images. It is been reported that the range of color space intensities for all ripeness stage is almost the same. In [9], researcher has analyzed RGB color model of FFB and classify it by using fuzzy logic. Image of fruitlets was captured indoor and they used image subtraction for background removal. This pre processing method is practical for indoor environment and its manage to give $86.67 \%$ accuracy. The work by[6] had taken the image of FFB in open space. In this discussion, there are analyzing MLP in ANN and founded that classifier will perform better with more than 3 hidden neurons in the hidden layer. In another work of them[10], trained the hidden and output neurons using Lavenberg Marquardt. In this work, it is reported that hue measurement has shown to be a good discriminator compared to RGB and CIEXY values. Another work for other fruits and vegetables also been discussed and reported. By [11], color analysis and back projection algorithm was used in order to extract the features of dates. Since this work needed consistent and uniform illumination, light source was designed and balance the variability in light source temperature. Besides other process, this work also stress on the process of image acquisition. For example, camera was mounted fixedly 40 $\mathrm{cm}$ above the dates. This is to give the consistencies of the image. In other work[12], has discussed how color map of images is utilized to mimic the manual visual assessment for detection of mango ripeness stage. This studies found that there have a significant changes in RGB color components and HSV along with ripeness stage. In other work, image processing, combined with pattern recognition has been applied in fruit sorting and classification to achieve high accuracy in [13]. In this work, bag of visual model was implemented to classify sweet and bitter almond. This method used to detect the appearance of the different almond classes. Studies reported that the key point detector is an important role in order to achieve high accuracy of detection and 
classification. In [14], researchers manage to classify fruit by using color and texture. This studies apply Gray-level Co-occurrence Matrix (GLCM) and statistical color features which extracted from the segmented image. The segmentation method is not only dependent on color but also dependent on edge information. Although there have a lot of studies done to determining the ripeness of fruits and vegetables, there are not manyresearch done for FFB ripeness stage in outdoor environment and classify it by fruit detach. The objective of this work is to investigate the ripeness of Fresh Fruit Bunch by using two way. They are with color features and bag of visual word. Color extraction by hue measurement was implemented in color features. Details of the process will be discussed in Section 3 of this paper. Algorithm were implemented using python and matlab and result for both method will be disscused in section 4 .

\section{METHODOLOGY}

There have two method which are implemented in this work. These two method are using color analysis and bag of visual words. Both two method start with same step which is image acquisition.

\section{Image acquisition.}

400 image of FFB was collected from plantation in North Malaysia. The image taken was under sunlight by smartphone camera which FFB still on the trees or FFB was gather in open space. FFB then are graded manually by human grader as soon as image taken. There have 4 ripeness stage involve in this grading: ripe, underripe, overripe and unripe. The ground truth of the image and condition from the human grader had been noted and recorded. Image then been resize and standardize $757 \times 568$ pixel dimension.

\section{a) Color Features Method}

\section{Pre Processing}

Dataset was created and split using SQLite3 in python path. A total of 360 images are used for training and 40 images used for testing. Image then cropped using python software and going through image segmentation process. Image segmentation is a process that divideimage into region. Its partitioning a digital image into multiple segments sets of pixels. In this process, image will be more meaningful and simple to analyze after it's been segmented. K-mean clustering algorithm been used to segmented the image in this work. Clustering is a method or an algorithm to classify or to bunch the objects based on its attributes and features into several $\mathrm{K}$ groups. This algorithm accepts input in the form of data without class labels. The method of gathering information into a cluster can be done by calculating the closest distance from a data to a centroid point.

In this work, k-mean segmentation used for remove fruits and its spike. Firstly, K-means model been trained by using training set. After tested to clustering it for several attempt, we decided that 2 cluster and 300 iteration is enough for clustering in this work. Original image then being applied with k-means.

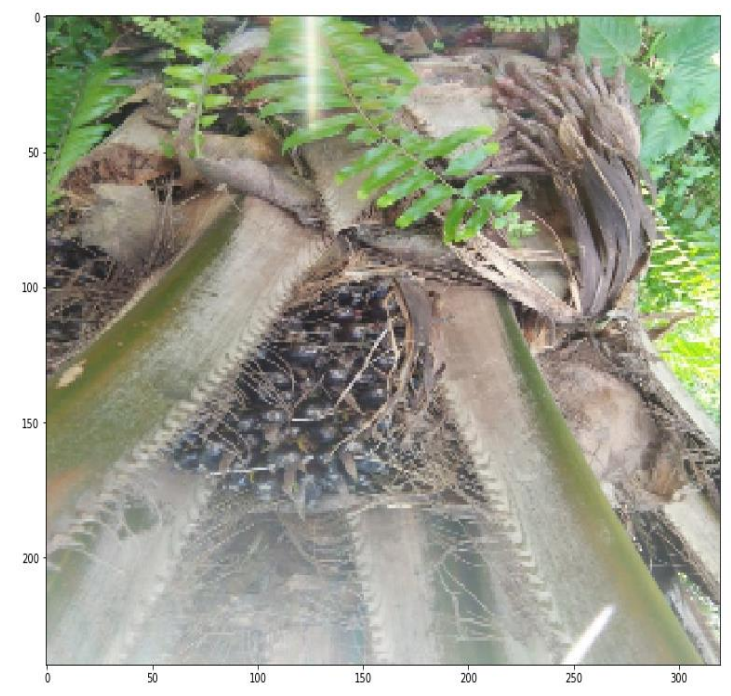

Figure 5. Original image

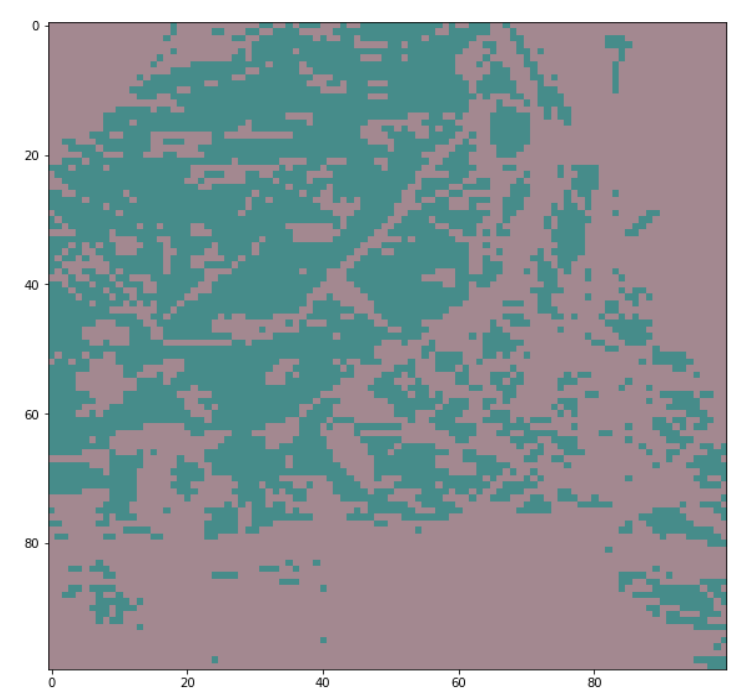

Figure 6. K-means training

After k-means was trained, image next was segmented.

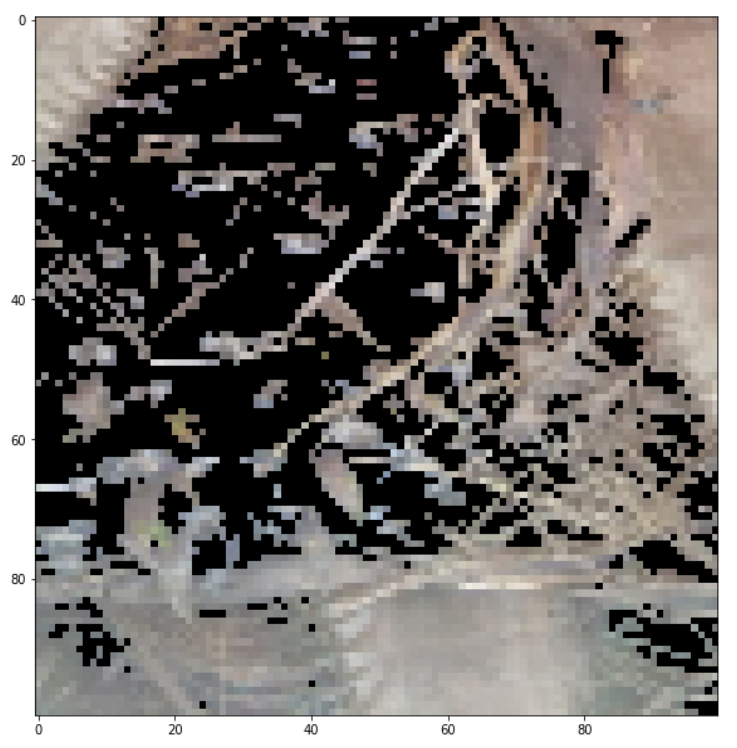

Figure 7. Segmented image 
Segmented image segment into fruit region. Image then was continue with feature extraction process

\section{Feature Extraction}

Feature extraction is a crucial step in determining the stage of ripeness. There have lot type of feature that can be extracted from an image such as texture, color, and size. In this work, color analysis had been used to determining the ripeness stage. This work are using hue measurement because as been reported in[10], compared with RGB or CIExy values, hue measurement has shown to be a good discriminator for oil palm fruits. Mathematically, the hue value $\mathrm{H}$ is given by this formula[15].

$$
\begin{gathered}
H=\cos ^{-1} \frac{\frac{1}{2}[(R-G)+(R-B)]}{\left[(R-G)^{2}+(R-B)(G-B)\right]^{\frac{1}{2}}} \text { if } B \leq G \\
H=360-\cos ^{-1} \frac{\frac{1}{2}[(R-G)+(R-B)]}{\left[(R-G)^{2}+(R-B)(G-B)\right]^{\frac{1}{2}}} \text { if } B \\
>G
\end{gathered}
$$

Where R, G and B isred, green and blue element in image. Hue histogram than being extracted from hue values.

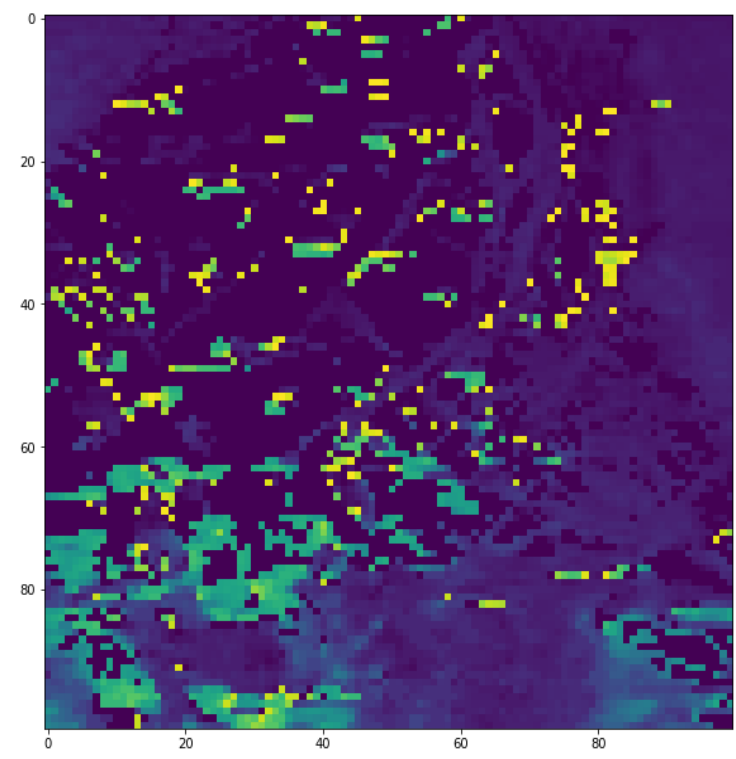

Figure 8. Color feature extraction

\section{Ripeness Clasification Using Support Vector System.}

The basic idea of Support Vector Machine is to maximise the hyperplane limit. It simply as an attempt to find the finest hyperplane that serves as a separator of two data classes within the input space. The best separator between the two classes can be found by measuring the margin of the hyperplane and finding its maximum point. Features of FFB that had been extracted in feature extraction procedure than used as input data in training process of SVM. LinearSvm was used. The accuracy of this method show $57 \%$ of accuracy for ripeness classification.

\section{a) Bag of visual words method}

Bag of Visual Word is an extention to the NLP algorithm used for image classification. It used widely in computer vision and classification research. Bag of Visual Model manage to recognize and detect the content of an image. Its follows by three simple step.

\section{Determination of image features}

In this step, 360 sample are used as training set, and 40 sample used as testing set. Image next was cropped and divided according to category of the ripeness. Features will be extract from both the training set. Since images do not actually contain discrete words, vocabulary of SURF features representative of eachimage classes had been construct. This essentially converts the image into a feature vector.

\section{Clustering}

Clustering is a method or an algorithm to classify or to group so that objects in the group are much similar than other group. BOVW used K-means Clustering as in clustering process. The goal of this K-Mean Clustering is to minimize the distance between each point. K-Means iterates over each of the input process and then relocate the cluster centroids. BOVW gives an encode for counting the visual word occurrences in an image. Histogram than was produced which become a new and reduced representation of an image.

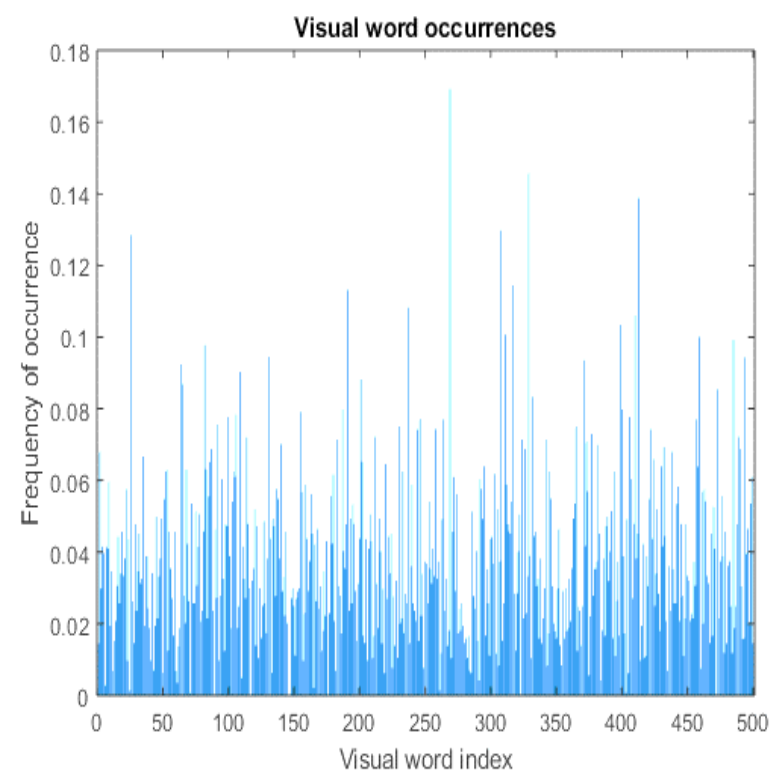

Figure 9. Histogram of visual word occurrences

\section{Training and testing}

Encoded training image then was inputted into train image classifier next to be utilized to formulate feature vector. The classifier contains the number of categories and the category labels for the input images. The function trains a support vector machine (SVM) multiclass classifier using the input bag. After being train, newly trained classifier next can be test on test image. 


\section{RESULT AND DISCUSSION}

a) Color features extraction

\begin{tabular}{|c|c|c|c|c|}
\hline \multirow{2}{*}{ KNOWN } & \multicolumn{4}{|c|}{ PREDICTED } \\
\cline { 2 - 5 } & OVERIPE & RIPE & UNDERIPE & UNRIPE \\
\hline OVERIPE & 5 & 3 & 0 & 2 \\
\hline RIPE & 2 & 7 & 1 & 0 \\
\hline UNDERIPE & 0 & 4 & 3 & 3 \\
\hline UNRIPE & 1 & 0 & 1 & 8 \\
\hline
\end{tabular}

Table 1. Confusion Matrix of classification for color features

Shown in table 1, was the confusion matrix for the test set for color features method. 40 image was used as test image. There is four possible predicted class which are 'overripe' for overripe FFB, 'ripe' for ripe FFB, 'underripe' for underripe FFB and 'unripe' for unripe FFB. Out of that 40 predictions, classifier predicts 5 overripe, 7 as ripe, 3 as underripe and 8 as unripe. In reality, 10 sample is the categories of each of the classes respectively. $57 \%$ is the accuracy of this classification.

a) Bag of Visual Words method

\begin{tabular}{|c|c|c|c|c|}
\hline \multirow{2}{*}{ KNOWN } & \multicolumn{4}{|c|}{ PREDICTED } \\
\cline { 2 - 5 } & OVERIPE & RIPE & UNDERIPE & UNRIPE \\
\hline OVERIPE & 4 & 3 & 1 & 2 \\
\hline RIPE & 0 & 10 & 0 & 0 \\
\hline UNDERIPE & 1 & 1 & 6 & 2 \\
\hline UNRIPE & 0 & 1 & 1 & 8 \\
\hline
\end{tabular}

Table 2. Confusion matrix for bag of visual word classification

In this paper, we get the result for both method and compare classification for FFB ripeness using color features with hue measurement as the indicator and bag of visual words method.

Shown in table 2, was the confusion matrix for the bag of visual word method experiment. There are also four possible predicted class which are 'overripe' for overripe FFB, 'ripe' for ripe FFB, 'underripe' for underripe FFB and 'unripe' for unripe FFB. The classifier made a total of 40 image for prediction. Out of those 40 prediction, classifier predict 4 as overripe, 10 as ripe, 6 as underripe and 8 as unripe. The average accuracy of this classifier is $70 \%$.

There also an error in predicting the result. 1 underripe FFB is being classify as overripe FFB. The classifier also confuse between ripe and overripe FF. Unripe FFB is being the highest error when 2 of it classified as underripe and 2 sample classify as overripe. Major observation of this result show that classifier majorly confuse between the increment stage of ripeness. This might be because there have a vagueness in keypoint detector.

Based on this result, bag of visual words give more accuracy than color features.

\section{CONCLUSION}

The experiment was conducted to classify the ripeness of FFB by implement the color features and BOVW model to see whether this method could be effective for ripeness stage of FFB both by color and texture. In the color feature experiment, , hue measurement was used as a features to determine the ripeness of FFB. Imagewas being process with image segmentation, color feature extraction and also training and testing with support vector machine. For BOVW experiment, the model cluster the image FFB, extract the features by using SURF descriptors, train and classify the ripeness stage of FFB. There have 500 features that had been extracted. In reality of palm oil industries, there have two main conditions in determining the ripeness of FFB which are based on the surface color and based on fruit detach. Future work should be investigated in both condition in order to make a good accuracy of classification. If good result achieve, its can be implemented in industry, so that it can help planter harvest oil palm fruit only at its optimum time. This will help to increase the productivity of palm oil production. It also can help reduce the yield gap of FFB oil in production.

However, in order to get that objective, future work using other method and algorithm such as SIFT and combining both color and texture features should be investigate thoroughly. Other methodwhich implementthe color analysis also should be testing in order to get better result.

\section{ACKNOWLEDGMENT}

The authors would like to thank Universiti Teknologi Malaysia and the Ministry of Higher Education for their supports. This project is supported by Research University Grant Vote Q.K130000.2409.04G18.

\section{REFERENCES}

[1] W. I. W. Ishak and R. M. Hudzari, "Image based modeling for oil palm fruit maturity prediction," $J$. Food Agric. Environ., vol. 8, no. 2, 1, pp. 469-476, 2010.

[2] Y. A. Tan, K. W. Low, C. K. Lee, and K. S. Low, "Imaging technique for quantification of oil palm fruit ripeness and oil content," Eur. J. Lipid Sci. Technol., vol. 112, no. 8, pp. 838-843, 2010.

[3] Z. B. Mat Sharif, N. B. Mohd Taib, M. S. Bin Yusof, M. Z. Bin Rahim, A. L. Bin Mohd Tobi, and M. S. Bin Othman, "Study on Handing Process and Quality Degradation of Oil Palm Fresh Fruit Bunches (FFB)," IOP Conf. Ser. Mater. Sci. Eng., vol. 203, no. 1, 2017.

[4] M. Makky and P. Soni, "Development of an automatic grading machine for oil palm fresh fruits bunches (FFBs) based on machine vision," Comput. Electron. Agric., vol. 93, pp. 129-139, 2013.

[5] Malaysian Palm Oil Board, "MANUAL PENGGREDAN BUAH KELAPA SAWIT," 2017. [Online]. Available: http://wskj.mpob.gov.my/pnp/gredffb.htm. 
[Accessed: 23-Jul-2018].

[6] N. Fadilah, J. Mohamad-Saleh, Z. A. Halim, H. Ibrahim, and S. S. S. Ali, "Intelligent color vision system for ripeness classification of oil palm fresh fruit bunch," Sensors (Switzerland), vol. 12, no. 10, pp. 14179-14195, 2012.

[7] T. Brosnan and D. Sun, "Inspection and grading of agricultural and food products by computer $\mathrm{v}$ ision systems * a re v iew," vol. 36, pp. 193-213, 2002.

[8] W. Ishak and W. Ismail, "Hue Optical Properties to Model Oil Palm Fresh Fruit Bunches Maturity Index," pp. 15-17, 2010.

[9] Z. May and M. H. Amaran, "Automated Oil Palm Fruit Grading System using Artificial Intelligence," Int. J. Video Image Process. Netw. Secur. IJVIPNSIJENS, vol. 11, no. June, pp. 30-35, 2011.

[10] N. Fadilah and J. Mohamad-Saleh, "Color feature extraction of oil palm fresh fruit bunch image for ripeness classification," 13th Int. Conf. Appl. Comput. Appl. Comput. Sci., pp. 51-55, 2014.

[11] D. Zhang, D. Lee, B. J. Tippetts, and K. D. Lillywhite, "Date maturity and quality evaluation using color distribution analysis and back projection," J. Food Eng., vol. 131, pp. 161-169, 2014.
[12] R. P. Salunkhel and A. A. Patif, "Image Processing for Mango Ripening Stage Detection: RGB and HSV method," pp. 362-365, 2015.

[13] A. Nasirahmadi and S. M. Ashtiani, "ScienceDirect Bag-of-Feature model for sweet and bitter almond classification," Biosyst. Eng., vol. 156, pp. 51-60, 2017.

[14] S. Jana, S. Basak, and R. Parekh, 2017 : Automatic Fruit Recognition from Natural Images using Color and Texture Features. 2017.

[15] M. Z. Abdullah, L. C. Guan, A. M. D. Mohamed, and M. A. M. Noor, "Color Vision System for Ripeness Inspection of," J. Food Process. Preserv., vol. 26, no. 604, pp. 213-235, 2002.

[13] A. Nasirahmadi and S. M. Ashtiani, "ScienceDirect Bag-of-Feature model for sweet and bitter almond classification," Biosyst. Eng., vol. 156, pp. 51-60, 2017.

[14] S. Jana, S. Basak, and R. Parekh, 2017 : Automatic Fruit Recognition from Natural Images using Color and Texture Features. 2017.

[15] M. Z. Abdullah, L. C. Guan, A. M. D. Mohamed, and M. A. M. Noor, "Color Vision System for Ripeness Inspection of,” J. Food Process. Preserv., vol. 26, no. 604, pp. 213-235, 2002. 